\title{
How we made the IGZO transistor
}

\author{
Thin-film transistors made from indium gallium zinc oxide (IGZO) are driving the next evolution in active-matrix \\ flat panel displays. Hideo Hosono recounts how demand for a high-performance alternative to amorphous silicon \\ transistors led to their development.
}

\section{Hideo Hosono}

iquid crystal displays (LCD) began to replace cathode-ray tube-based televisions in the early 2000s. The pixels in these LCDs were, until 2012, driven exclusively by thinfilm transistors (TFTs) that use amorphous hydrogenated silicon (a-Si:H) as the semiconducting channel. But as demand grew for ever larger displays, with better viewing and energy performance, a need for improved TFTs emerged. TFTs block light coming from the display, so they need to be small, which lowers their current-driving ability. Additionally, there was a push to move away from LCD technology and into organic light emitting diodes (OLEDs) in order to capitalize on their better performance, which includes direct emission and richer colours, but OLEDs need to be driven by large amounts of current, at levels a-Si:H TFTs cannot accommodate. What was needed was a new technology that could be used to create TFTs with a high charge carrier mobility, which could drive large currents.

Charge carrier mobility tells us how quickly charge carriers (electrons and holes) can move through a semiconductor, and determines the amount of current we can drive through a TFT of a given size. This is limited by the nature of the path that charge carriers take though a material. This path can be thought of in terms of the atomic orbitals and how they interconnect to make up the conduction and valence bands - the energetic levels at which the electrons and holes are free to move around. The $s p^{3}$ hybridized orbitals in silicon are highly directional, and in the crystalline state the silicon-silicon bonds properly overlap, providing an efficient path to travel through. However, in amorphous silicon, structural disorder leads to disconnects and conduction cannot occur through the orbitals in the same way. Instead, the charge carriers have to hop across the orbitals, which leads to mobilities of at most $1 \mathrm{~cm}^{2} \mathrm{~V}^{-1} \mathrm{~s}^{-1}$ - about 2-3 orders of magnitude lower than in crystalline silicon.

I thought an ionic semiconductor with a wide bandgap might be different because the conduction and valence bands are made up of orbitals that have different characteristics. In certain oxide materials containing $p$-block metal cations, the vacant metal $s$-orbital that predominantly makes up the conduction band minimum is spherical and spatially

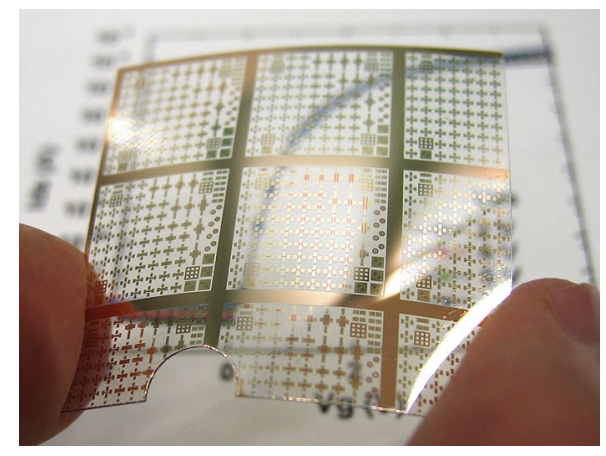

Fig= 1 | Amorphous indium gallium zinc oxide thinfilm transistors fabricated on a plastic substrate. Credit: Hideya Kumomi

spread, making their overlap insensitive to bonding angle variation. As such, I hoped that when going from their ordered crystalline state to their disordered amorphous state, these oxide semiconductors might still show a high mobility. To verify this hypothesis, I, together with my research group, selected and sputtered a range of metal oxide thin films, including $\mathrm{CdO}-\mathrm{GeO}_{2}$. Testing their Hall mobility, these amorphous thin films showed values higher than $10 \mathrm{~cm}^{2} \mathrm{~V}^{-1} \mathrm{~s}^{-1}$, which is comparable to polycrystalline thin films. The idea and experimental results were published in the May issue of the Journal of Non-Crystalline Solids in 1996, but gained little interest from the research community. By 2004, for example, the paper had received only 4 citations, and 2 of those were self-citations!

After a few years verifying the validity of the hypothesis, we started research on transparent oxide TFTs. To develop high-mobility TFTs using transparent conductive oxides, the major issue was their high carrier concentrations (the TFTs can't be turned off). Indium oxide is a typical transparent conductive oxide with a large mobility, but reducing its excessive carrier concentration is difficult due the ease with which oxygen vacancies form. We tested $\mathrm{InGaO}_{3}(\mathrm{ZnO})_{m}$ epitaxial films, where $m=4$ and 5 , as I believed the unique local structure around $\mathrm{Ga}^{3+}$ would help lower the carrier concentration. As expected, the carrier concentration in epitaxial $\mathrm{InGaO}_{3}(\mathrm{ZnO})_{4}$ reduced to a suitable level, and when applied in TFTs showed excellent performance, with a mobility of approximately $80 \mathrm{~cm}^{2}$ $\mathrm{V}^{-1} \mathrm{~s}^{-1}$. We then fabricated thin films using amorphous $\mathrm{InGaZnO}_{4}$ (a-IGZO). The electron concentrations in the resulting a-IGZO thin films, which were deposited on glass substrates at room temperature, were below $10^{16} \mathrm{~cm}^{-3}$ and the TFTs exhibited mobilities of approximately $10 \mathrm{~cm}^{2} \mathrm{~V}^{-1} \mathrm{~s}^{-1}$ - an order of magnitude larger than that of a-Si:H TFTs. We also found that TFTs fabricated on plastic substrates offered almost identical performance (Fig. 1). At this point we applied for patents and then submitted our paper to Nature, which was published in 2004.

Demonstrations of IGZO TFT-based displays began to emerge in 2007, when Samsung Electronics first reported a-IGZO TFT-based high-definition LCDs. Demonstrations of IGZO TFT-based backplane LCDs in smart phones and tablet computers then appeared around 2012. Arguably the most striking application emerged in 2015, when LG Display demonstrated large-sized OLED televisions. Here the a-IGZO TFT was indispensable because of the need for high mobility and simple formation over large areas.

I believe the success of IGZO transistors is due to a number of factors. First is their high mobility, which is an order of magnitude higher than a-Si:H, and the excellent controllability of their carrier concentration. Second, the production process is almost the same as a-Si:H, except for the deposition of the channel layer (chemical vapour deposition is used for a-Si:H whereas sputtering is used for IGZO), making integration into current manufacturing workflows relatively painless. Third, synthesizing large-sized high-quality sputtering targets is relatively easy, which also makes thin-film deposition on largesized substrates a relatively simple task. With these attributes, IGZO transistors provide a promising backplane technology to drive the future of flat panel displays.

\section{Hideo Hosono \\ Tokyo Institute of Technology, Yokohama, Japan. e-mail:hosono@msl.titech.ac.jp}

Published online: 13 July 2018 https://doi.org/10.1038/s41928-018-0106-0 